This information is current as of April 26, 2023.

\title{
MR Imaging Differences in the Circle of Willis between Healthy Children and Adults
}

K.P. Guilliams, N. Gupta, S. Srinivasan, M.M. Binkley, C. Ying, L. Couture, J. Gross, A. Wallace, R.C. McKinstry, K. Vo, J.-M. Lee, H. An and M.S. Goyal

AJNR Am J Neuroradiol 2021, 42 (11) 2062-2069

doi: https://doi.org/10.3174/ajnr.A7290

http://www.ajnr.org/content/42/11/2062 


\title{
MR Imaging Differences in the Circle of Willis between Healthy Children and Adults
}

\author{
(D).P. Guilliams, (D) N. Gupta, (D). Srinivasan, (D) M.M. Binkley, (DC. Ying, L. Couture, (D). Gross, (D)A. Wallace, (D) R.C. McKinstry,
} (D) K. Vo, (D).-M. Lee, (D) H. An, and (D)M.S. Goyal O.

\begin{abstract}
BACKGROUND AND PURPOSE: Asymmetries in the circle of Willis have been associated with several conditions, including migraines and stroke, but they may also be age-dependent. This study examined the impact of age and age-dependent changes in cerebral perfusion on circle of Willis anatomy in healthy children and adults.
\end{abstract}

MATERIALS AND METHODS: We performed an observational, cross-sectional study of bright and black-blood imaging of the proximal cerebral vasculature using TOF-MRA and T2 sampling perfection with application-optimized contrasts by using different flip angle evolution (T2-SPACE) imaging at the level of the circle of Willis in 23 healthy children and 43 healthy adults (4-74 years of age). We compared arterial diameters measured manually and cerebral perfusion via pseudocontinuous arterial spin-labeling between children and adults.

RESULTS: We found that the summed cross-sectional area of the circle of Willis is larger in children than in adults, though the effect size was smaller with T2-SPACE-based measurements than with TOF-MRA. The circle of Willis is also more symmetric in children, and nonvisualized segments occur more frequently in adults than in children. Moreover, the size and symmetry of the circle of Willis correlate with cerebral perfusion.

CONCLUSIONS: Our results demonstrate that the circle of Willis is different in size and symmetry in healthy children compared with adults, likely associated with developmental changes in cerebral perfusion. Further work is needed to understand why asymmetric vasculature develops in some but not all adults.

ABBREVIATIONS: AcomA = anterior communicating artery; CoW = circle of Willis; CoW-area $=$ overall CoW size; CoW-di $=$ CoW deviation index; ICC = intraclass correlation coefficient; PCASL = pseudocontinuous arterial spin-labeling; PcomA = posterior communicating artery

$F^{\mathrm{i} i}$ rst described by Thomas Willis in the 1600 s, $^{1}$ the circle of Willis $(\mathrm{CoW})$ is a vascular ring that functionally lies between the distal ICAs and basilar arteries, and the proximal anterior, middle, and posterior cerebral arteries (Fig 1). A complete CoW is often viewed as a mechanism to provide protective collateral flow. Asymmetries in the CoW may be associated with increased stroke risk, particularly in people with carotid stenosis ${ }^{2-4}$ or aneurysms. ${ }^{5}$ Recent studies have noted an increase in asymmetries with age, ${ }^{6,7}$ but these studies have been limited to adults, with most adults reported older than 40 years of age.

Received January 29, 2021; accepted after revision July 19.

From the Department of Neurology (K.P.G., M.M.B., J.-M.L., M.S.G.), Department of Pediatrics (K.P.G., R.C.M.), Mallinckrodt Institute of Radiology (K.P.G., N.G., S.S.,

C.Y., L.C., R.C.M., K.V., J.-M.L., H.A., M.S.G.), Departments of Biomedical Engineering (J.-M.L.), and Neuroscience (M.S.G.), Washington University School of Medicine, St Louis, Missouri; Division of Neuroradiology (J.G.), Midwest Radiology, St. Paul, Minnesota; and Department of Neurointerventional Surgery (A.W.), Ascension Columbia St. Mary's Hospital, Milwaukee, Wisconsin.

K.P. Guilliams and N. Gupta contributed equally to this work.

This work is supported by the National Institutes of Health (R01AG057536, MSG; K23NS099472, KPG), the McDonnell Center for Systems Neuroscience (M.S.G), and unrestricted funds from the Mallinckrodt Institute of Radiology (M.S.G.).
In detailed postmortem studies since 1905, completely absent segments of the CoW are rare, on the order of $\sim 3 \%-5 \% .{ }^{8-10}$ However, asymmetries of the CoW due to hypoplastic or "functionally absent" segments are much more common, with estimates of approximately $30 \%-60 \%$ in adults. ${ }^{8,9,11-13}$ Hypoplastic segments are also common with older age in a heterogeneous clinical population of older adults. ${ }^{6,7,14}$ However, despite $>3$ decades of MRA use, there are presently scant normative CoW imaging data in children. Studies of the CoW in children are limited

Author Contributions: K.P.G.: data acquisition, analysis and interpretation, drafted manuscript; N.G., S.S., J.G., C.Y., L.C.: data acquisition and analysis, critical review of manuscript; M.M.B.: data analysis and interpretation and critical review of manuscript; A.W.: study design, data collection, and critical review of manuscript; R.C.M., K.V., J.-M.L., H.A.: data interpretation and critical review of manuscript; M.S.G.: study design, data collection, analysis and interpretation, drafted manuscript. All authors reviewed and approved the final version of this manuscript.

Please address correspondence to M.S. Goyal, MD, Washington University School of Medicine, 510 South Kingshighway Blvd, St. Louis, MO 63110; e-mail: goyalm@wustl.edu

- Indicates open access to non-subscribers at www.ajnr.org

Indicates article with online supplemental data.

http://dx.doi.org/10.3174/ajnr.A7290 


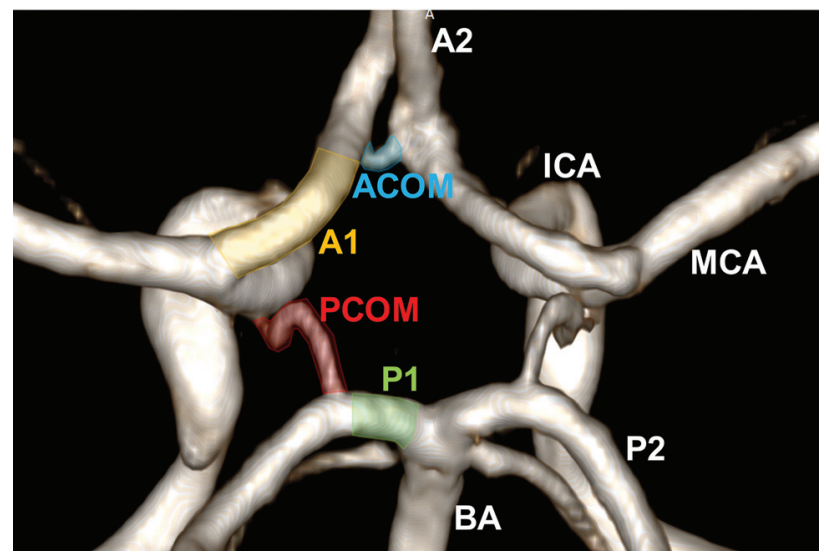

FIG 1. The circle of Willis. 3D volume-rendering of the CoW from a TOF-MRA in a young child. This shows the key segments comprising the vascular ring, including AcomA (blue), the first segment of the anterior cerebral artery ( $\mathrm{Al}$, orange), the PcomA (red), and the first segment of the posterior cerebral artery ( $\mathrm{Pl}$, green). Other arteries feeding the CoW (the ICA and the basilar artery [BA]) and supplied by the CoW (second segment of the anterior cerebral artery [A2], MCA, and second segment of the posterior cerebral artery [P2]) are also labeled.

to a postmortem study ${ }^{15}$ and to those with neuropathology, including brain tumors treated with radiation, ${ }^{16}$ neurofibromatosis, ${ }^{17}$ neonatal stroke, ${ }^{18}$ and prematurity. ${ }^{19}$ There remains a need for evaluation of the CoW in healthy children in comparison with adults.

The CoW is particularly unique with respect to the possible vascular effects of cerebral perfusion changes because it represents a redundancy in proximal cerebral circulation. ${ }^{1}$ Cerebral perfusion and CBF peak in the first decade of life and then decrease substantially both globally and in a regionally specific manner. ${ }^{20-24}$ Local CBF is, in part, regulated by arteriolar resistance, though capillaries and large cerebral arteries may also contribute to overall cerebral vascular resistance. ${ }^{25-27}$ Moreover, high CBF implies either higher blood velocity, larger arterial cross-section area, or both throughout the cerebral vasculature. ${ }^{28,29}$ Yet currently, the effects of $\mathrm{CBF}$, particularly higher $\mathrm{CBF}$ in childhood, on the cerebral vasculature are unknown.

The objective of our study was to evaluate the size and topology of the CoW across the life span in healthy children compared with healthy adults using both TOF-MRA and confirmatory T2 sampling perfection with application-optimized contrasts by using different flip angle evolution (SPACE sequence; Siemens) techniques and to investigate possible CBF mechanisms of CoW changes. We hypothesized that the CoW would be larger and more symmetric/complete in healthy children than in adults, primarily in association with increased $\mathrm{CBF}$ during childhood.

\section{MATERIALS AND METHODS \\ Subjects}

This study was approved by the institutional review board of Washington University School of Medicine and performed according to provisions within the Declaration of Helsinki. Self-described healthy participants 4-79years of age were recruited via flyers, word of mouth, and a volunteer data base maintained by our institution. Specific exclusion criteria included any known neurologic or vascular illness or contraindications to MR imaging. In accordance with Strengthening the Reporting of Observational Studies in Epidemiology guidelines for observational cross-sectional studies, we attempted to balance the number of subjects across 3 age groups (children: 4-18, younger adults: 19-40, and older adults: 4180 years of age) by sex and for them to remain representative of the ethnic composition of the greater St. Louis area to minimize bias.

\section{MR Imaging and Angiography}

Recruited participants underwent up to 30 minutes of MR imaging on either a $3 \mathrm{~T}$ Tim Trio (Siemens) scanner $(n=58)$, or on the same scanner following an upgrade to a 3T Magnetom PrismaFit (Siemens) scanner $(n=8)$, both with a 32 -channel head coil. MR imaging protocol specifics are provided in the Online Supplemental Data. Our goal was to produce a protocol that had short-enough sequences to minimize motion artifacts, particularly in young children, yet that could be equally applied to all age groups in this cohort to avoid sequence-specific biases. Participants all underwent the following imaging: 1) isotropic $1-\mathrm{mm}^{3}$ sagittal T1-MPRAGE; 2) $3 \mathrm{D}$ TOF-MRA with a low flip angle $\left(18^{\circ}\right.$, to minimize intraluminal saturation effects) centered over the CoW with $0.57 \times 0.57 \times$ $0.69 \mathrm{~cm}$ resolution; 3) 3D T2-SPACE centered over the CoW with $0.53 \times 0.53 \times 0.50 \mathrm{~cm}$ resolution; and 4) a pseudocontinuous arterial spin-labeling (pCASL) sequence $(\mathrm{TE} / \mathrm{TR}=12 / 3780 \mathrm{~ms}$, resolution $=3.0 \times 3.0 \times 5.0 \mathrm{~mm}$, labeling duration $=2000 \mathrm{~ms}$, postlabeling delay $=1500 \mathrm{~ms}$, background suppression not performed). An additional fast inversion recovery sequence of the superior sagittal sinus was obtained to estimate individual blood T1 values for CBF calculations. A subset of participants $(n=36)$ also underwent a phase-contrast sequence of the ICAs and vertebral arteries at the level of the $\mathrm{C} 2-\mathrm{C} 3$ disk space to quantify whole-brain blood flow $(\mathrm{TE} / \mathrm{TR}=4.06 / 107.2 \mathrm{~ms}$, resolution $=0.7 \times 0.7 \times$ $5.0 \mathrm{~mm}$, flip angle $=25^{\circ}, 10$ averages, velocity encoding $=120$ $\mathrm{cm} / \mathrm{s}$ ). Reader 1 (N.G.) reviewed all imaging for excessive noise, motion artifacts, or incomplete imaging.

\section{Vessel Segment Nomenclature}

Definition and nomenclature of the vessel segments comprising the CoW and major cerebral arteries follow current clinical convention (Fig 1). On the basis of prior modeling work, ${ }^{30}$ we applied a slightly simplified definition of the CoW as comprising 7 segments: bilateral A1, P1, and PcomA segments, and a single anterior communicating artery (AcomA). The ICA termini and basilar apex are defined as inflow segments, and the M1, A2, and P2 segments, as outflow segments, and neither are included in the simplified CoW definition. The ICA segments are labeled from $\mathrm{C} 1$ proximally in the upper neck through $\mathrm{C} 7$ at the terminus, according to Bouthillier et al, ${ }^{31}$ in 1996.

\section{Vessel Diameter Measurement}

Vessel measurements were performed on both TOF-MRA and T2-SPACE sequences independently. The source TOF-MRA and T2-SPACE images were analyzed using RadiAnt DICOM Viewer software (Version 4 or 5; Medixant). A board-certified neuroradiologist (M.S.G., with >3years of postfellowship experience) trained and supervised reader 1 (N.G., an undergraduate student) to perform vessel-diameter measurements. Measurements were 


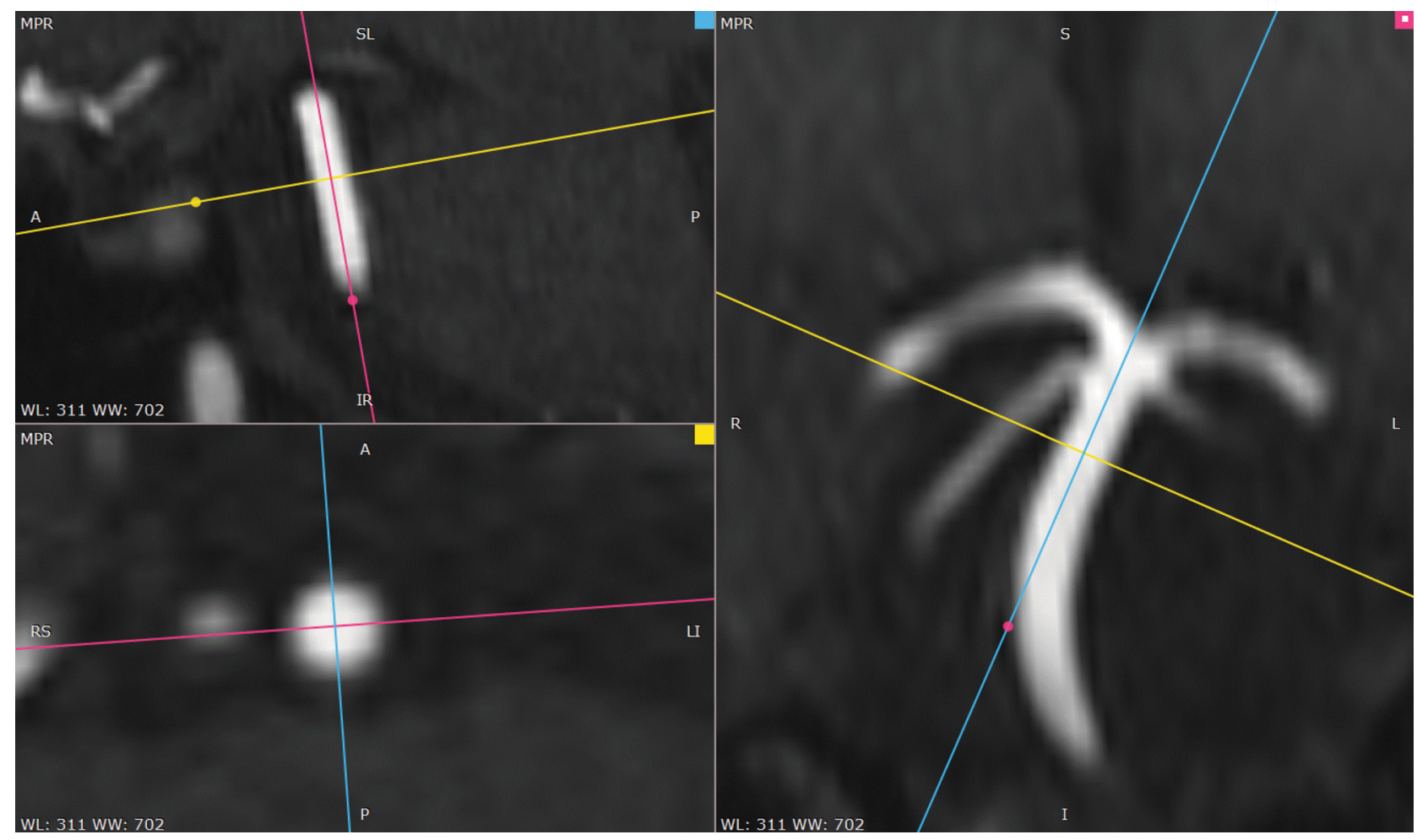

FIG 2. Double-oblique multiplanar reconstruction for vessel-diameter measurement. Two orthogonal planes of reconstruction were aligned along the long axis of each arterial segment to be measured. These were then used to construct a cross-sectional plane through the midportion of the vessel segment on which 2 perpendicular diameters were measured and averaged. Note that partial volume effects at the margins of the artery could result in a slight degree of measurement bias and error. WL indicates window level; WW, window width.

performed using the RadiAnt default window settings to avoid windowing bias. Measurements on all vessel segments from a subset of participants $(n=15)$ were repeated by both reader 1 and reader 2 (J.G., a neuroradiology fellow) to assess intra- and interrater reliability and to confirm the accuracy of measurements by reader 1 .

The diameters of C7, distal basilar artery, M1, M2, A1, A2, P1, and $\mathrm{P} 2$ segments, AcomA, PcomA, and superior cerebellar arteries were measured on both the TOF-MRA and T2-SPACE images. On the TOF-MRA, the readers made additional measurements of the ICA segments C1-C5/C6, the proximal and mid-basilar artery, anterior inferior cerebellar artery, PICA, and vertebral arteries because the TOF-MRA sequence had a larger craniocaudal FOV. The procedure for measurement included multiplanar reconstruction with the double-oblique technique to identify the mid-crosssection orthogonal to the course of each vessel segment being measured (Fig 2). Care was taken to define the edge of a vessel consistently due to partial volume effects. Readers measured 2 perpendicular diameters at this cross-section using the ruler tool in RadiAnt and then averaged the 2 to produce a final diameter. Nonvisualized segments were assigned a value of zero.

\section{Topologic Analysis of the CoW}

We assessed the total summed cross-sectional area of the 7 measured segments of the CoW to determine its overall size (CoWarea). To quantify asymmetries and grade differences between hypoplastic and missing segments while also remaining indifferent to which segments caused asymmetries, we reduced the CoW topology a priori to a single variable defined as the CoW deviation index (CoW-di). We calculated CoW-di as follows: First, we normalized each segment diameter to the mean diameter from all 7 segments within the same CoW. Next, we created a cohort-average CoW, defined as the set of mean segment ratios derived from all participants, children and adults. Finally, a CoW-di for each individual was calculated as the total Euclidean distance between the 7 segment ratios of the individual CoW and the cohort-averaged CoW (Fig 3). A low CoW-di indicates that the relative sizes of the CoW segments are similar to those of the cohort-averaged CoW, whereas a high CoW-di indicates deviation from the group, either due to higher degree of anterior-posterior and/or left-right asymmetry, including that arising from nonvisualized segments (which were assigned a zero diameter).

\section{Cerebral Perfusion Analysis}

The pCASL data were first preprocessed using a uniform pipeline that included the following: 1) skull stripping with the FSL Brain Extraction Tool (Version 6.0; http://fsl.fmrib.ox.ac.uk/fsl/fslwiki/ BET); 2) motion correction using MCFLIRT from FSL (https:// fsl.fmrib.ox.ac.uk/fsl/fslwiki/MCFLIRT), and 3) spatial smoothing using in-house Matlab code (MathWorks). CBF was then calculated from pCASL using Equation $1 .^{32}$

$$
\text { 1) } f=\frac{\lambda \Delta M R_{1 a}}{2 \alpha M_{0}\left[\exp \left(-w R_{1 a}\right)-\exp \left(-(\tau+w) R_{1 a}\right]\right.},
$$

where $f$ is $\mathrm{CBF}, \lambda(0.9 \mathrm{~g} / \mathrm{mL})$ is the blood/tissue water partition coefficient, $R_{1 \mathrm{a}}$ (1/blood T1 value) is the longitudinal relaxation 


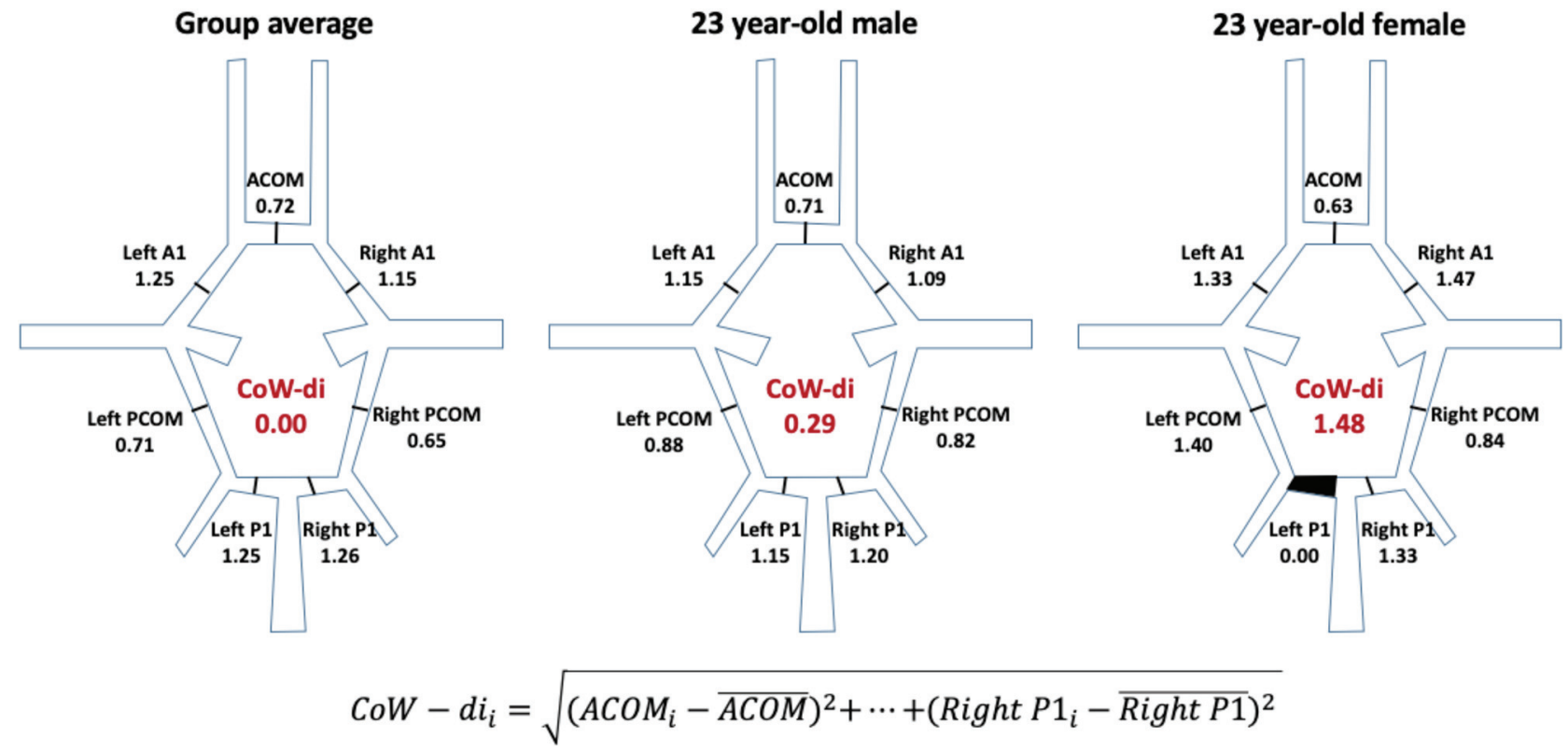

FIG 3. Measurement of the CoW-di. A group average of the ratio of each CoW segment to the mean CoW diameter was calculated. For each individual CoW, similar ratios were calculated for each of the 7 segments. The CoW-di was then calculated as the Euclidean distance between the individual CoW ratios and the group-average ratios, thus representing the degree to which an individual CoW deviated in size from the group average. Examples are shown for two 23 -year-old individuals, one with a very low CoW-di (0.29) and another with a high CoW-di (1.48), largely due to a nonvisualized left Pl segment.

rate of blood, $\alpha$ is the tagging efficiency, $M_{0}$ is the equilibrium magnetization of brain, $\tau$ is the duration of the labeling pulse, and $w$ is the postlabeling delay time. We assigned blood T1 values by age group after measuring them manually from the fast inversion recovery sequence in 56 individuals and averaging them by ages (4$11,12-20,21-40,41-60,61-80$ years of age). The resulting CBF maps were then summarized to ROIs defined by the DesikanKilliany and subcortical atlases following registration to individual MPRAGE sequences that were processed by FreeSurfer Version 5.3 (http://surfer.nmr.mgh.harvard.edu) as discussed further below.

\section{Whole-Brain Blood Flow Quantitation}

We obtained phase-contrast MR imaging sequences to measure blood velocities and cross-section areas of the distal cervical ICAs and vertebral arteries in a subset of individuals. These were measured using the Vitrea software (Version 6.9.2; Vital Images) semi-automated flow-quantification tool within the cardiac flow program. Flow within each vessel was summed to compute whole-brain blood flow.

\section{Brain Structure Volumetric Analysis}

Individual MPRAGE image volumes were analyzed with FreeSurfer, Version 5.3 (default options), to provide estimates of intracranial volume, whole-brain volume, gray and white matter volumes, ventricular volumes, and regional cortical and subcortical volumes. Cortical segmentation was performed according to the DesikanKilliany atlas.

\section{Statistical Analysis}

Statistical analysis was performed using the statistical programming language $\mathrm{R}$ statistical and computing software, Version 3.5.2 and above (http://www.r-project.org/) and SAS software
(Version 9.4; SAS Institute). Despite a priori hypotheses that the CoW would be larger and more symmetric in children, $P$ values are provided for these comparisons first from the conservative 2tailed $t$ test for initial analysis with TOF-MRA measurements and then the 1-tailed $t$ test for confirmatory analysis with the T2SPACE measurements because the directionality of difference is already known. The Mann-Whitney $U$ (Wilcoxon rank sum) test was also performed when there was significant skew in the distributions. Descriptive statistics, confidence intervals, and additional specific statistical tests are detailed in the Results section.

\section{Data Availability}

Individual numeric measurements and raw imaging data obtained in this study will be provided on reasonable request.

\section{RESULTS}

A total of 66 participants underwent MR imaging in this study, ranging from 4 to 74 years of age (children: $4-18$ years, $n=23$; younger adults: $19-40$ years, $n=23$; older adults: $41-74$ years, $n=20$ ), including a similar number of males and females (34 males, 32 females). Five participants were excluded due to qualitatively noisy or incomplete TOF data, leaving data from 61 participants (19 children, 42 adults) for further analysis. Of these 61 participants, 7 had qualitatively noisy or incomplete T2-SPACE data (4 children, 3 adults) that was not included in subsequent analysis.

\section{Intra- and Interrater Reliability of Manual Vessel Diameter Measurements}

Vessel diameter measurements (68 total measurements across both sequences) were repeated for a subset comprising 15 participants ( $\sim 1000$ total measurements) twice by reader 1 and once by 

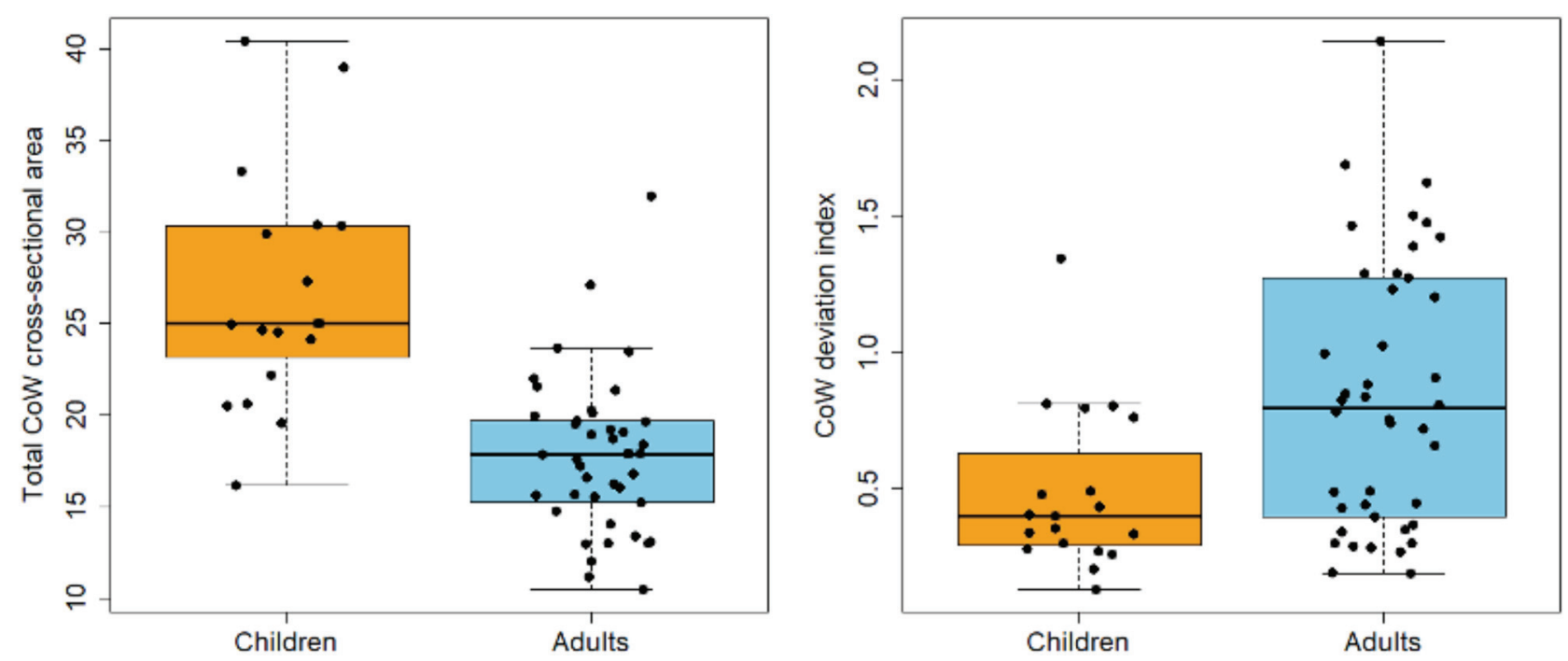

FIG 4. Differences in CoW size and symmetry in children versus adults. The total CoW cross-sectional area was measured in healthy children $(n=19)$ and adults $(n=42)$ on TOF-MRA images as described in the text. The mean total CoW cross-sectional area was larger in children than in adults (children's mean CoW-area $=27.7 \mathrm{~mm}^{2}$ versus adults' mean CoW-area $=17.8 \mathrm{~mm}^{2}, P<.0001$ ). Note that T2-SPACE measurements replicated these results, though with a smaller effect size as discussed in the text. CoW topology in children also more closely resembled the average topology found in the whole group, despite there being more adults in the whole group (children's mean CoW-di $=0.48$ versus adults' mean CoW-di $=0.84, P<.001$ ). As described in the text, the difference in CoW topology is, in part, due to a higher prevalence of nonvisualized CoW segments in adults.

reader 2. Across participants, the intrarater reliability was excellent (intraclass correlation coefficient $[$ ICC $]=0.96$, range $=0.89-0.98$ across individual participants) with low bias and mean absolute deviation (mean bias $=0.02 \mathrm{~mm}$, mean absolute deviation = $0.216 \mathrm{~mm}$ ). Interrater reliability was also excellent $(\mathrm{ICC}=0.86$, range $=0.79-0.92$ ), but there was more bias (mean bias= $0.49 \mathrm{~mm}$ ); after correcting for this bias, the mean absolute deviation was low (mean absolute deviation $=0.31 \mathrm{~mm}$ ), suggesting a systematic cause for the bias. After we reviewed the measurement procedure with each reader, this bias likely occurred due to differences in how each reader accounted for partial volume effects at the margins of the vessel lumen. When comparing the mean diameter measurements of C7, M1, and the distal basilar artery with those published recently for a large cohort of adults, the measurements of reader 1 (N.G.) are closer to previously published radii (published: $\mathrm{C} 7=1.7 \mathrm{~mm}, \mathrm{M} 1=1.1 \mathrm{~mm}$, distal basilar $=1.3 \mathrm{~mm}^{33}$; reader one: $1.7,1.3,1.6 \mathrm{~mm}$; reader two: 2.0, 1.6, $1.7 \mathrm{~mm}$, respectively). Reader 1 also completed measurements in all participants. Primary measurements by reader 1 are, accordingly, used for the remainder of the analysis.

\section{TOF-MRA versus T2-SPACE Measurements}

The intra- and interrater reliabilities across all participant data were similar when calculated independently for the TOF-MRA and T2SPACE measurements (TOF-MRA: intrarater ICC $=0.91$, interrater $\mathrm{ICC}=0.89$; T2-SPACE: intrarater $\mathrm{ICC}=0.93$, interrater $\mathrm{ICC}=0.90$ ). The mean difference between the TOF-MRA and T2SPACE measurements was $0.147 \mathrm{~mm}$ (interquartile range $=0.126-$ $0.168 \mathrm{~mm}$ ) with limits of agreement ranging from -0.582 to $0.876 \mathrm{~mm}$. Although the overall difference between TOF-MRA and T2-SPACE (TOF-MRA measurements were on average larger) was statistically significant (paired $t$ test, $P<.001$ ), vessel diameter did not influence the difference between TOF-MRA and T2-SPACE because proportional bias was not significant (slope $=0.0003$, $P=.984$ ). Further analysis of the circle of Willis did, however, reveal an age effect on the differences between TOF-MRA and T2SPACE on the A1 and P1 segments bilaterally (Pearson $r=-0.33$ to -0.70 , all $P<.05$ ), but not the PcomA and AcomA segments ( $r=-0.25$ to -0.08 , all $P>.05$ ), suggesting that age-related CBF differences may mediate differences between TOF-MRA and T2SPACE.

\section{CoW Size and Topology in Children versus Adults}

The total cross-sectional area of the CoW based on TOF-MRA was, on average, 56\% larger in children than in adults (CoWarea: children mean $=27.7 \mathrm{~mm}^{2}$ [95\% CI, 24.1-31.2] versus adults mean $=17.8 \mathrm{~mm}^{2}$ [95\% CI, 16.5-19.1]; $t$ test, $P<.001$ ) (Fig $4 A$ and Table). With the T2-SPACE data, the CoW was, on average, $17 \%$ larger in children $(\mathrm{CoW}$-area: children mean $=16.6$ $\mathrm{mm}^{2}\left[14.5-18.7 \mathrm{~mm}^{2}\right]$ versus adults' mean $=14.2 \mathrm{~mm}^{2}[13.0-$ $\left.15.5 \mathrm{~mm}^{2}\right]$; $t$ test, $\left.P<.05\right)$. CoW topology also differed between children and adults. Children had a lower CoW-di, reflecting greater symmetry, than adults with both the TOF-MRA measurements (CoW-di: children's mean $=0.48$ [95\% CI, 0.35-0.62] versus adults' mean $=0.84$ [95\% CI, 0.69-0.99]; Mann-Whitney $U$ test, $P<.01)($ Fig $3 B$ ) and T2-SPACE data (CoW-di: children's mean $=0.68[0.53-0.83]$ versus adults' mean $=0.93[0.77-1.08]$; $P<.05$, Mann-Whitney $U$ test, $P<.05$ ). Among 133 potential CoW segments in 19 children, 5 were nonvisualized among 5 children (3.8\% of vessel segments, 3 PcomAs and 2 AcomAs; $26 \%$ of the 19 children), whereas among 294 potential CoW segments in 42 adults, 31 were nonvisualized among 23 adults (10.5\% of vessel segments, 2 A1s, 18 PcomAs, 4 P1s, and 7 AcomAs; $55 \%$ of the 42 adults). The odds of a nonvisualized 
Summary CoW measurements in healthy children versus adults

\begin{tabular}{lcc}
\hline & $\begin{array}{c}\text { Children (0-18 years of } \\
\text { age) }\end{array}$ & $\begin{array}{c}\text { Adults (19-74 years of } \\
\text { age) }\end{array}$ \\
\hline $\begin{array}{l}\text { No. of participants } \\
\text { Age (range) (yr) }\end{array}$ & 23 & 43 \\
Sex (female, male) & $9.5(4-18)$ & $40.7(19-74)$ \\
Excluded data & 11,12 & 21,22 \\
TOF-MRA CoW-area (mean) & 4 of 23 & 1 of 43 \\
$\quad\left(\right.$ mm $\left.^{2}\right)$ & $27.7(\mathrm{SD}, 7.9)$ & $17.8(\mathrm{SD}, 4.2)$ \\
TOF-MRA CoW-di (mean) & $0.48(\mathrm{SD}, 0.30)$ & $0.84(\mathrm{SD}, 0.49)$ \\
T2-SPACE CoW-area (mean) & $16.6(\mathrm{SD}, 4.1)$ & $14.2(\mathrm{SD}, 4.0)$ \\
$\quad\left(\right.$ mm $\left.^{2}\right)$ & & \\
T2-SPACE CoW-di (mean) & $0.68(\mathrm{SD}, 0.29)$ & $0.93(\mathrm{SD}, 0.49)$ \\
Nonvisualized segments & $5 / 133(3.8 \%)$ & $31 / 294(10.5 \%)$ \\
Whole-brain CBF (mean) (L/min) & $1.10(\mathrm{SD}, 0.24)$ & $0.69(\mathrm{SD}, 0.21)$ \\
\hline
\end{tabular}

$r=0.84 ; 95 \%$ CI, $0.70-0.91$; linear model slope $=0.96$; $95 \% \mathrm{CI}, 0.75-$ 1.17), suggesting that the methods used here to quantify $\mathrm{CBF}$ with pCASL are reasonably accurate and unbiased. All subsequent results are thus with the pCASL data, which were available for more of the participants.

Whole-brain CBF decreased significantly with age $(n=53$, Pearson $r=$ $-0.71 ; 95 \% \mathrm{CI},-0.54$ to -0.82 ) and was, on average, $59 \%$ higher in children (children's mean whole-brain $\mathrm{CBF}=1.10 \mathrm{~L} / \mathrm{min}(n=16)$ versus adults' mean $=0.69 \mathrm{~L} / \mathrm{min}[n=38] ; t$

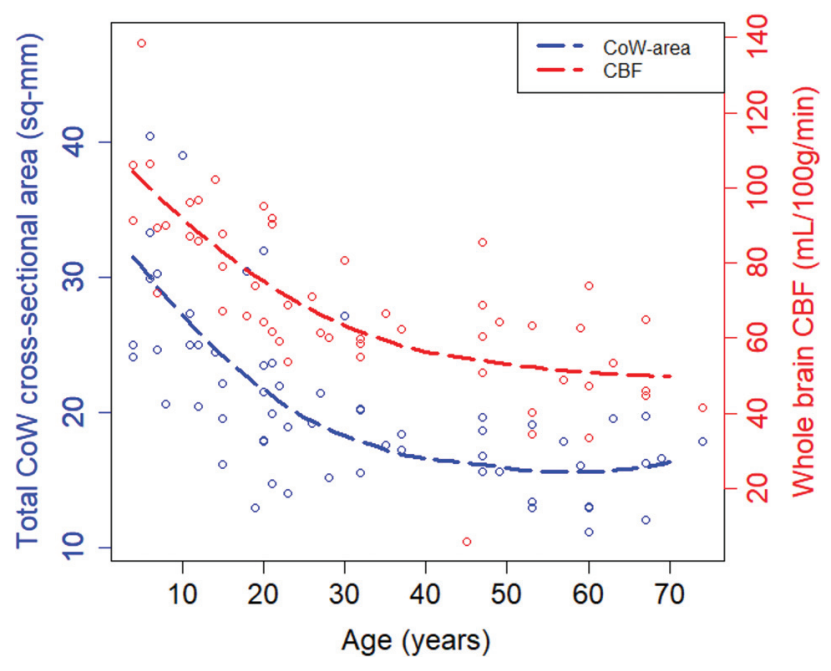

FIG 5. CoW size tracks with CBF changes during development. Total CoW cross-sectional area based on TOF-MRA and whole-brain CBF was measured in participants ranging from 4 to 74 years of age. There is a gradual decrease in whole-brain CBF from childhood to adulthood that parallels the decrease in total CoW cross-sectional area. Across participants, CoW size also correlated with whole-brain CBF (Pearson $r=0.68$, $\left.P<10^{-7}\right)$. Smoothing lines are for visualization purposes and are based on LOcally WEighted Scatter-plot Smoother fits with span $=1$.

segment were thus lower in children compared with adults (Fisher exact test, $\mathrm{OR}=0.36$; 95\% CI, 0.11-0.96; $P<.05$ ).

\section{Relationship among CoW Segments}

In an exploratory analysis of CoW segment-to-segment relationships across all ages, PcomA diameter showed a strong inverse relationship with the ipsilateral P1 diameter (Pearson $r=-0.67 \&$ $-0.71, \mathrm{~L} \& \mathrm{R})$, as expected. Most interesting, whereas there was a modest inverse correlation between the right A1 and AcomA segments $(r=-0.52)$, there was minimal correlation between the left A1 and AcomA segment $(r=-0.05)$. Further analysis of other CoW data sets is needed to determine whether this asymmetry in A1-AcomA size relationship is generalizable to other cohorts.

\section{CoW Size and Topology in Relation to Cerebral Perfusion}

Whole-brain blood flow estimated using pCASL correlated highly and with minimal bias compared with that measured using phase-contrast MR imaging ( $n=37$ paired comparisons, Pearson test, $P<.00001)$. Based on the TOF-MRA data, the size and topology of the CoW correlate with whole-brain CBF (CoW-area $\sim \mathrm{CBF}$ : Pearson $r=0.68$; 95\% CI, 0.50-0.80; CoW-di $\sim \mathrm{CBF}: r=$ $-0.41 ; 95 \% \mathrm{CI},-0.16$ to -0.61$)$. Across the age span, decreases in TOF-MRA-based measurements of the CoW-area tracked closely with decreases in CBF (Fig 5), and the proportional differences between children and adults are similar for CoW-area (56\%) and whole-brain CBF (59\%).

To further explore the relationship between CoW topology and $\mathrm{CBF}$, we compared individual diameter asymmetry in PcomA and A1 size with asymmetry in the posterior cerebral artery and anterior cerebral artery territory $\mathrm{CBF}$, respectively, the latter defined on the basis of anatomic ROIs typically within those arterial territories (posterior cerebral artery = cuneus, pericalcarine, lateral occipital regions; anterior cerebral artery = rostral and caudal anterior cingulate, medial orbitofrontal, and superior-frontal). PcomA vessel-size asymmetry correlated modestly with posterior cerebral artery territory CBF asymmetry (Pearson $r=0.39$; 95\% CI, 0.13-0.59). However, there was no correlation between A1 vessel size asymmetry and anterior cerebral artery territory CBF asymmetry (Pearson $r=0.03$ ).

\section{DISCUSSION}

Since the anatomic and functional exposition of the CoW by Thomas Willis in the 1600s, ${ }^{1}$ numerous studies of the CoW have been published in a variety of research domains. The CoW is often viewed in the context of providing protective collateral flow to the cerebral arteries in case of flow restriction within the internal carotid or basilar arteries. The importance of this protection is currently most evident in older patients with atherosclerotic carotid or vertebrobasilar occlusive disease. The CoW may also protect against direct compression of the carotid artery in the neck and thereby reduce the effects of choking or neck traumaa role that might have been more evolutionarily pertinent.

Yet, the CoW may also have physiologic roles beyond providing protective collateral circulation. ${ }^{34}$ The current study suggests that the CoW is both larger and more visibly complete in healthy children than in adults; this feature is associated with developmental differences in CBF. It is possible, then, that the CoW also provides a developmental function, namely in adjudicating flow between the anterior and posterior circulations during normal brain development and maturation. During early infant brain 
development, metabolism is higher in the occipital lobes and thal$\mathrm{ami}^{35}{ }^{35}$ which are predominantly supplied by the posterior cerebral arteries and branches arising from the P1 and PcomA segments. As the brain matures, brain metabolism and blood flow increase in other parts of the brain-most prominently in the medial and prefrontal regions..$^{21,23,36}$ Thus, there is an overall developmental shift from the posterior-to-anterior circulationpredominant CBF. Although changes in carotid or basilar artery size could accommodate such changes, the CoW is well-positioned to assist in this transition. Indeed, prenatal cerebrovascular development is associated with additional anastomoses between the carotid and vertebrobasilar circulation that typically regress before birth. ${ }^{37,38}$ The CoW might represent a later stage of cerebrovascular development, though with limited regression that occurs only in some adults.

Our finding that whole-brain $\mathrm{CBF}$ is associated with $\mathrm{CoW}$ size and topology is consistent with the relationship between flow and the product of cross-sectional area and blood velocity. The relationship between cerebral artery size and blood flow is further well-established in the context of arteriovenous malformation, in which the feeding arteries are typically much enlarged and then might normalize following obliteration of the arteriovenous malformation. Our study did not include blood-velocity measurements in the CoW. MR imaging-based velocity measurements in the A1 segment do identify mildly higher velocities in children compared with adults, though the difference in 1 study was small. ${ }^{33}$ Such newer MR imaging-based flow-quantification methods as well as the transcranial Doppler method would help to fill in this gap and determine whether changes in CBF are also associated with changes in blood velocity within the CoW. ${ }^{39,40}$

Nonvisualized segments in the CoW were notably rare in children (3.8\% of all vessel segments) and similar to that reported in postmortem series $(\sim 3 \%-5 \%)$. Nonvisualized segments were more common in adults (10.5\%), likely due to the overall smaller size of the CoW and decreased CBF that occurs in normal aging. This finding suggests that studies of functionally absent or hypoplastic CoW segments in this context should include measures of both age and CBF as a potential mediator of their findings. Our proportion of adults with nonvisualized segments (55\%) is comparable with other studies in adults, though it was less frequent than in a recently reported population-based study suggesting that up to $88 \%$ of adults have a nonvisualized segment; ${ }^{7}$ however, this study exclusively examined adults 40-90years of age, whereas half of our adult population was younger than 40 years of age, and we did not include any adults older than 80 years. Our study was also purposefully biased toward healthy individuals. Further work is needed to understand why the older population has decreased CBF and increased asymmetries and whether these might be a result of normal decreasing CBF and metabolic changes in the brain, pathologic changes in the brain such as cerebrovascular disease, or some combination of these factors.

An important strength of our study was the replication of key findings with both a bright-blood, flow-sensitive sequence (TOFMRA) and a black-blood, anatomic sequence (T2-SPACE). Both sequences demonstrated the CoW to be larger and more symmetric in children than in adults. However, the effect size was considerably larger with TOF-MRA-based measurements than with T2-
SPACE (eg, 56\% versus 17\% difference in CoW-area). Because differences between TOF-MRA- and T2-SPACE-based arterial diameters were, in part, related to the participant's age, we propose that some of this difference might arise from differences in cerebral blood flow exaggerating flow-related enhancement and thereby the apparent vessel diameters in children/adults on the TOF-MRA images. Our findings, thus, prescribe caution when using only TOF-MRA or T2-SPACE to determine vessel diameters, particularly if $\mathrm{CBF}$ is expected to vary; applying $>1$ sequence with different underlying assumptions and artifacts may allow convergent findings.

There are important limitations to the current study. First, whereas the diameter measurements showed excellent intrarater reliability, there was a small systematic interrater bias in these measurements that was likely due to resolution limitations. Future improvements in MR imaging technology and sequence development will likely result in higher resolution methods that provide higher precision and accuracy. Also, whereas these data provide initial measures of the CoW in healthy children in comparison with adults, use of these measures as a normative control for other studies should be avoided because data limitations include reader- and sequence-specific biases, the modest number of children in this study, and a cohort representative of only a single institution and its surrounding community.

\section{CONCLUSIONS}

We found that the CoW is larger and more symmetric in children, associated with developmental and aging changes in CBF. Our study suggests that further investigation is warranted into how and why asymmetries arise in the CoW during typical aging or in the context of disease.

Disclosures: Kristin P. Guilliams—UNRELATED: Expert Testimony: Grant, National Institutes of Health*, Comments: expert witness for neonatal stroke (2019, 2020). Adam Wallace-UNRELATED: Expert Testimony: Hallberg Law, PA, Comments: expert testimony provided for malpractice suit. Jin-Moo Lee-RELATED: Grant: National Institutes of Health*; UNRELATED: Consultancy: Regnera Medical, Comments: consulting on a stroke-recovery drug; Grants/Grants Pending: Biogen, Comments: funded a study on stroke genetics.* Hongyu An-UNRELATED: Consultancy: Pfizer; Grants/Grants Pending: Siemens. Manu Goyal—RELATED: Grant: McDonnell Center for Systems Neuroscience, National Institutes of Health/National Institute of Aging*; UNRELATED: Grants/Grants Pending: National Institutes of Health*; Stock/Stock Options: IBM; Travel/Accommodations/Meeting Expenses Unrelated to Activities Listed: Capital Medical University, Shandong Madic Technology, Tancheng Talent Office, Comments: honoraria and travel expenses for attending and speaking at the 2019 Linyi Brain PET Conference and Xuanwu Hospital. *Money paid to the institution.

\section{REFERENCES}

1. Willis T. Cerebri Anatome: Cui Accessit Nervorum Descriptio Et Usus. 1663 https://archive.org/details/cerebrianatomecu00will. Accessed April 8,2020

2. Hoksbergen AW, Legemate DA, Csiba L, et al. Absent collateral function of the circle of Willis as risk factor for ischemic stroke. Cerebrovasc Dis 2003;16:191-98 CrossRef Medline

3. Kim KM, Kang HS, Lee WJ, et al. Clinical significance of the circle of Willis in intracranial atherosclerotic stenosis. J Neurointerv Surg 2016;8:251-55 CrossRef Medline

4. Banga PV, Varga A, Csobay-Novak C, et al. Incomplete circle of Willis is associated with a higher incidence of neurologic events during carotid eversion endarterectomy without shunting. $J$ Vasc Surg 2018;68:1764-71 CrossRef Medline 
5. Lazzaro MA, Ouyang B, Chen M. The role of circle of Willis anomalies in cerebral aneurysm rupture. J Neurointerv Surg 2012;4:22-26 CrossRef Medline

6. Eaton RG, Shah VS, Dornbos D, 3rd, et al. Demographic age-related variation in circle of Willis completeness assessed by digital subtraction angiography. Brain Circ 2020;6:31-37 CrossRef Medline

7. Hindenes LB, Haberg AK, Johnsen LH, et al. Variations in the circle of Willis in a large population sample using 3D TOF angiography: the Tromso Study. PLoS One 2020;15:e0241373 CrossRef Medline

8. Kapoor K, Singh B, Dewan LI. Variations in the configuration of the circle of Willis. Anat Sci Int 008;83:96-106 CrossRef Medline

9. Alpers BJ, Berry RG, Paddison RM. Anatomical studies of the circle of Willis in normal brain. AMA Arch Neurol Psychiatry 1959;81:40918 CrossRef Medline

10. Fawcett E, Blachford JV. The circle of Willis: an examination of 700 specimens. J Anat Physiol 1905;40:63(Pt 1):2-70 Medline

11. Krabbe-Hartkamp MJ, van der Grond J, de Leeuw FE, et al. Circle of Willis: morphologic variation on three-dimensional time-of-flight MR angiograms. Radiology 1998;207:103-11 CrossRef Medline

12. Hendrikse J, van Raamt AF, van der Graaf Y, et al. Distribution of cerebral blood flow in the circle of Willis. Radiology 2005;235:18489 CrossRef Medline

13. Klimek-Piotrowska W, Rybicka M, Wojnarska A, et al. A multitude of variations in the configuration of the circle of Willis: an autopsy study. Anat Sci Int 2016;91:325-33 CrossRef Medline

14. Zaninovich OA, Ramey WL, Walter CM, et al. Completion of the circle of Willis varies by gender, age, and indication for computed tomography angiography. World Neurosurg 2017;106:953-63 CrossRef Medline

15. Piechna A, Lombarski L, Krajewski P, et al. Age dependence of critical pressure in the segments and branches of the circle of Willis. Neurol Neurochir Pol 2017;51:146-48 CrossRef Medline

16. El-Fayech C, Haddy N, Allodji RS, et al. Cerebrovascular diseases in childhood cancer survivors: role of the radiation dose to Willis circle arteries. Int J Radiat Oncol Biol Phys 2017;97:278-86 CrossRef Medline

17. Bekiesińska-Figatowska M, Braôgoszewska H, Duczkowski M, et al. Circle of Willis abnormalities in children with neurofibromatosis type 1. Neurol Neurochir Pol 2014;48:15-20 CrossRef Medline

18. Husson B, Hertz-Pannier L, Adamsbaum C, et al. MR angiography findings in infants with neonatal arterial ischemic stroke in the middle cerebral artery territory: a prospective study using circle of Willis MR angiography. Eur J Radiol 2016;85:1329-35 CrossRef Medline

19. Malamateniou C, Adams ME, Srinivasan L, et al. The anatomic variations of the circle of Willis in preterm-at-term and term-born infants: an MR angiography study at 3T. AJNR Am J Neuroradiol 2009;30:1955-62 CrossRef Medline

20. Kennedy C, Sokoloff L. An adaptation of the nitrous oxide method to the study of the cerebral circulation in children; normal values for cerebral blood flow and cerebral metabolic rate in childhood. $J$ Clin Invest 1957;36:1130-07 CrossRef Medline

21. Chiron C, Raynaud C, Maziere B, et al. Changes in regional cerebral blood flow during brain maturation in children and adolescents. $J$ Nucl Med 1992;33:696-703 Medline
22. Avants BB, Duda JT, Kilroy E, et al. The pediatric template of brain perfusion. Sci Data 2015;2:150003 CrossRef Medline

23. Satterthwaite TD, Shinohara RT, Wolf DH, et al. Impact of puberty on the evolution of cerebral perfusion during adolescence. Proc Natl Acad Sci U S A 2014;111:8643-48 CrossRef Medline

24. Goyal MS, Hawrylycz M, Miller JA, et al. Aerobic glycolysis in the human brain is associated with development and neotenous gene expression. Cell Metab 2014;19:49-57 CrossRef Medline

25. Faraci FM, Heistad DD. Regulation of large cerebral arteries and cerebral microvascular pressure. Circ Res 1990;66:8-17 CrossRef Medline

26. Heistad DD, Marcus ML, Abboud FM. Role of large arteries in regulation of cerebral blood flow in dogs. J Clin Invest 1978;62:761-68 CrossRef Medline

27. Willie CK, Tzeng YC, Fisher JA, et al. Integrative regulation of human brain blood flow. J Physiol 2014;592:841-59 CrossRef Medline

28. Sacks AH, Tickner EG. The compressibility of blood. Biorheology 1968;5:271-74 CrossRef Medline

29. Wang SH, Lee LP, Lee JS. A linear relation between the compressibility and density of blood. J Acoust Soc Am 2001;109:390-96 CrossRef Medline

30. Devault K, Gremaud PA, Novak V, et al. Blood flow in the circle of Willis: modeling and calibration. Multiscale Model Simul 2008;7:888909 CrossRef Medline

31. Bouthillier A, van Loveren HR, Keller JT. Segments of the internal carotid artery: a new classification. Neurosurgery 1996;38:425-32; discussion 432-33 CrossRef Medline

32. Wang J, Zhang Y, Wolf RL, et al. Amplitude-modulated continuous arterial spin-labeling 3.0-T perfusion MR imaging with a single coil: feasibility study. Radiology 2005;235:218-28 CrossRef Medline

33. Mouches $P$, Forkert ND. A statistical atlas of cerebral arteries generated using multi-center MRA datasets from healthy subjects. $S c i$ Data 2019;6:29 A CrossRef Medline

34. Vrselja Z, Brkic H, Mrdenovic S, et al. Function of circle of Willis. J Cereb Blood Flow Metab 2014;34:578-84 CrossRef Medline

35. Goyal MS, Raichle ME. Glucose requirements of the developing human brain. J Pediatr Gastroenterol Nutr 2018;66(Suppl 3):S46-49 CrossRef Medline

36. Chugani HT, Phelps ME, Mazziotta JC. Positron emission tomography study of human brain functional development. Ann Neurol 1987;22:487-97 CrossRef Medline

37. Menshawi K, Mohr JP, Gutierrez J. A functional perspective on the embryology and anatomy of the cerebral blood supply. J Stroke 2015;17:144-58 CrossRef Medline

38. Raybaud C. Normal and abnormal embryology and development of the intracranial vascular system. Neurosurg Clin N Am 2010;21:399426 CrossRef Medline

39. Wu C, Honarmand AR, Schnell S, et al. Age-related changes of normal cerebral and cardiac blood flow in children and adults aged 7 months to 61 years. J Am Heart Assoc. 2016;5:e002657 CrossRef Medline

40. Garcia J, Barker AJ, Murphy I, et al. Four-dimensional flow magnetic resonance imaging-based characterization of aortic morphometry and haemodynamics: impact of age, aortic diameter, and valve morphology. Eur Heart J Cardiovasc Imaging 2016;17:877-84. Aug CrossRef Medline 